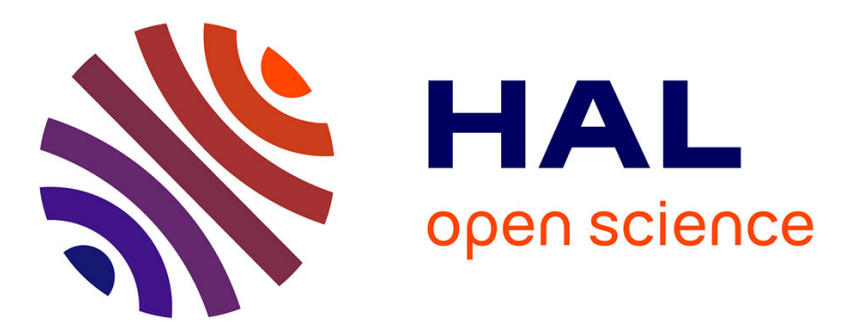

\title{
Neutron specific heat in the crust of neutron stars from the nuclear band theory
}

Nicolas Chamel, Jérôme Margueron, Elias Khan

\section{To cite this version:}

Nicolas Chamel, Jérôme Margueron, Elias Khan. Neutron specific heat in the crust of neutron stars from the nuclear band theory. Physical Review C, 2009, 79, pp.012801. 10.1103/PhysRevC.79.012801 . hal-00349072

\section{HAL Id: hal-00349072 https://hal.science/hal-00349072}

Submitted on 23 Dec 2008

HAL is a multi-disciplinary open access archive for the deposit and dissemination of scientific research documents, whether they are published or not. The documents may come from teaching and research institutions in France or abroad, or from public or private research centers.
L'archive ouverte pluridisciplinaire HAL, est destinée au dépôt et à la diffusion de documents scientifiques de niveau recherche, publiés ou non, émanant des établissements d'enseignement et de recherche français ou étrangers, des laboratoires publics ou privés. 


\title{
Neutron specific heat in the crust of neutron stars from the nuclear band theory
}

\author{
N. Chamel \\ Institut d'Astronomie et d'Astrophysique, Université Libre de Bruxelles, \\ CP226, Boulevard du Triomphe, 1050 Brussels, Belgium \\ J. Margueron and E. Khan \\ Institut de Physique Nucléaire, IN2P3-CNRS and Université Paris-Sud, F-91406 Orsay Cedex, France
}

(Dated: December 23, 2008)

\begin{abstract}
The inner crust of neutron stars, formed of a crystal lattice of nuclear clusters immersed in a sea of unbound neutrons, may be the unique example of periodic nuclear systems. We have calculated the neutron specific heat in the shallow part of the crust using the band theory of solids with Skyrme nucleon-nucleon interactions. We have also tested the validity of various approximations. We have found that the neutron specific heat is well described by that of a Fermi gas, while the motion of the unbound neutrons is strongly affected by the nuclear lattice. These apparently contradictory results are explained by the particular properties of the neutron Fermi surface.
\end{abstract}

PACS numbers:

Keywords: neutron star crust, neutron star cooling, band theory, specific heat, dynamical effective mass

Neutron stars are born in the gravitational core collapse of massive stars. With about one or twice the mass of the Sun compressed inside a radius of only 10 kilometers or so, neutron stars are among the most compact objects in the Universe [1]. The outer layers of the star, at densities below the neutron drip threshold $\rho_{\mathrm{ND}} \simeq 4 \times 10^{11}$ g. $\mathrm{cm}^{-3}$, are formed of a solid Coulomb lattice of neutron rich nuclei coexisting with a degenerate gas of relativistic electrons. The inner crust, at density above $\rho_{\mathrm{ND}}$ and below the crust-core transition density, which is at about half the density $\rho_{0}=2.8 \times 10^{14}$ g.cm ${ }^{-3}$ inside atomic nuclei, is permeated by a sea of unbound neutrons, which could be superfluid in some layers [2].

Many observed neutron star phenomena are intimately related to the physics of the crust. For instance, X-ray bursts in Low-Mass X-ray Binaries are associated with thermonuclear burning in the accreting neutron star envelope [3]. In the subgroup of soft X-ray transients, accretion outbursts are followed by long period of quiescence during which the accretion rate is essentially zero. In some cases, the period of accretion can last long enough for the crust to be heated out of equilibrium with the core. The thermal relaxation during the quiescent state has been recently monitored for KS 1731-260 and for MXB 1659-29 after an accretion episode of 12.5 and 2.5 years respectively [5]. These observations thus provide information on the thermal properties of neutron star crusts 6, 7]. The thermal relaxation of the crust could also be potentially observed in very young isolated neutron stars, $10-100$ years after their formation [8, 9].

The diffusion of heat in the inner crust is mainly governed by the thermal properties of the shallow layers, owing to their very low thermal diffusivity [9]. One of the key parameters is the neutron specific heat. In cooling simulations of neutron stars, the neutron specific heat is generally approximated by that of uniform neutron matter. At low enough temperatures, neutrons are predicted to become superfluid but the exact value of the critical temperature $T_{c}$ still remains uncertain [2]. Moreover, the presence of nuclear clusters is likely to modify the critical temperature. For instance, in Ref. [4], it has been shown that the pairing field in the dilute neutron gas completely vanishes just after the drip point.

The effects of superfluidity on the neutron specific heat are usually incorporated in the following way [10]:

$$
c_{V}^{\text {paired }}(T)=\mathcal{R}\left(T / T_{c}\right) c_{V}(T)
$$

where the renormalisation factor $\mathcal{R}\left(T / T_{c}\right)$ accounts for the effects of superfluidity and $c_{V}(T)$ stands for the specific heat calculated in the absence of superfluidity. In the above expression, the presence of the nuclear clusters is ignored. Their effects have been studied by solving the Hartree-Fock-Bogoliubov equations for the nucleons [11, 12] and by including the contribution of collective modes [13]. These calculations have been carried out within the spherical Wigner-Seitz approximation. However the validity of this approximation has been recently discussed 14. Pointing out the analogy between unbound neutrons in neutron star crusts and conduction electrons in ordinary metals, the band theory of solids have been applied and adapted to the nuclear scale 15, 16. It has shown for the first time the large effects of Bragg scattering on the motion of the unbound neutrons. One may expect that thermodynamical properties like the specific heat could also be modified.

In this paper, we investigate the effects of the solid crust on the neutron specific heat, within the framework of the nuclear band theory (Sec. II). Given the present uncertainties in the pairing problem, even in the simpler case of pure neutron matter [2], we will focus on the normal part $c_{V}(T)$ only. Results in the shallow layers are discussed in Sec. III and are compared to several approximations used in the literature. 


\section{A MICROSCOPIC MODEL OF THE NEUTRON STAR CRUST}

Following the standard assumptions, we consider a body centered cubic crystal with only one nuclear cluster per lattice site 11. We have taken the composition $(N, Z)$ of the clusters calculated by Negele and Vautherin [17. But the nucleon distributions have been recalculated at each temperature $T$ by solving the finite temperature Hartree-Fock equations with the Skyrme SLy4 effective nucleon-nucleon interaction [18]. Typical temperatures of interest for cooling isolated neutron stars and for accreting neutron stars lie in the range between $10^{7}$ to few $10^{9} \mathrm{~K}[9]$.

Fully self-consistent calculations within the band theory are computationally very expensive. We have thus solved the Hartree-Fock equations in two steps [14. First, we have determined self-consistently the nucleon distributions and mean fields in the Wigner-Seitz approximation with mixed Neumann-Dirichlet boundary conditions. We have removed the spurious fluctuations of the neutron density by averaging in the interstitial region 17]. In a second stage, we have solved the Schrödinger equation with Bloch boundary conditions using these self-consistent mean fields. We have fixed the lattice spacing so that the volume of the WignerSeitz polyhedron is equal to the volume of the spherical Wigner-Seitz cell of radius $R_{\text {cell }}$. We have employed the Linearized Augmented Plane Wave method described in details in Ref. [16]. As shown in Ref. [14], the one-body spin-orbit potential has a negligible effect on the neutron level ordering and was therefore neglected.

The specific heat (per unit volume) is defined as

$$
c_{V}(T)=\left.\frac{\partial U}{\partial T}\right|_{V}=\left.T \frac{\partial S}{\partial T}\right|_{V}
$$

(taking the Boltzmann's constant $k_{\mathrm{B}}=1$ ) where $U$ is the total internal energy density, $S$ the entropy density and the partial derivatives are evaluated at constant volume $V$. We have found that the latter expression is numerically more convenient. The entropy density of the

TABLE I: For the three shallow layers considered in this paper, are given: the total density $(\rho)$, the number of protons and neutrons $(Z, N$ respectively), the nuclear mass number $A$ (at $T=0)$, the radius of the cell $\left(R_{\text {cell }}\right)$ and the neutron gas density at $T=0\left(\rho_{n}^{G}\right)$.

\begin{tabular}{cccccc}
\hline \hline$\rho\left[\mathrm{g} . \mathrm{cm}^{-3}\right]$ & $Z$ & $N$ & $A$ & $R_{\text {cell }}[\mathrm{fm}]$ & $\rho_{n}^{G}\left[\mathrm{fm}^{-3}\right]$ \\
\hline $6.69 \times 10^{11}$ & 40 & 160 & 133 & 49.24 & $1.310^{-4}$ \\
$1.00 \times 10^{12}$ & 40 & 210 & 141 & 46.33 & $2.610^{-4}$ \\
$1.47 \times 10^{12}$ & 40 & 280 & 140 & 44.30 & $4.910^{-4}$ \\
\hline \hline
\end{tabular}

unbound neutrons is given by

$$
S=-\sum_{\alpha} \int \frac{\mathrm{d}^{3} \boldsymbol{k}}{\left(2 \pi^{3}\right)}\left[f_{\alpha \boldsymbol{k}} \ln f_{\alpha \boldsymbol{k}}+\left(1-f_{\alpha \boldsymbol{k}}\right) \ln \left(1-f_{\alpha \boldsymbol{k}}\right)\right]
$$

where $\alpha$ is the band index, $\boldsymbol{k}$ is the Bloch wave vector and $f_{\alpha \boldsymbol{k}}$ is the Fermi-Dirac distribution. The latter depends on the temperature and the neutron chemical potential $\mu_{n}$ which is determined from the total density $\rho_{n}^{G}$ of unbound neutrons by

$$
\rho_{n}^{G}=\sum_{\alpha} \int \frac{\mathrm{d}^{3} \boldsymbol{k}}{\left(2 \pi^{3}\right)} f_{\alpha \boldsymbol{k}}
$$

Integrations have been carried out using the special point method (see [16]). Note that the number of unbound neutrons per cluster is determined by the shape of the mean fields, which vary with temperature. Consequently $\rho_{n}^{G}$ can also depend on the temperature. In particular, with increasing temperature the most loosely bound neutrons may be excited into the delocalized states.

\section{RESULTS AND DISCUSSIONS}

We have considered three different layers in the shallow region of the inner crust. Their properties are summarized in Table I. The neutron chemical potential $\mu_{n}(T)$ and the entropy density $S(T)$, have been calculated for a set of temperatures between $T=10 \mathrm{keV}$ and $T=100$ $\mathrm{keV}$. We have then evaluated the specific heat according to Eq. (2), by interpolating the entropy density $S(T)$ with cubic splines and numerically differentiating.

For the range of temperatures considered here, $T \ll$ $\varepsilon_{\mathrm{F}}$, where $\varepsilon_{\mathrm{F}}$ is the Fermi energy. The specific heat is then approximately given by (ref. [19], p47)

$$
c_{V}(T) \simeq \frac{\pi^{2}}{3} g\left(\varepsilon_{\mathrm{F}}\right) T
$$

where $g\left(\varepsilon_{\mathrm{F}}\right)$ is the density of states at the Fermi level. This expression is valid provided i) the density of unbound neutrons $\rho_{n}^{G}$ and $\varepsilon_{\mathrm{F}}$ are independent of the temperature, ii) the density of states $g(\varepsilon)$ is sufficiently smooth. Pertaining to the first point, we have found numerically that $\rho_{n}^{G}$ varies very little so that the neutron evaporation/condensation phenomenon mentionned in Sect. If can be ignored. In a previous paper, we have shown that the density of states in the shallow layers of the crust is far from being smooth at a scale of few $\mathrm{keV}$ (see Fig. 6 of Ref. 14]). However, since we consider thermal energies of order $10 \mathrm{keV}$ or more, the variations of the density of states with the energy are expected to be much smaller at this scale as noticed in Ref. [14]. As can be seen in Fig. 1, the neutron specific heat varies almost linearly with $T$ as expected from Eq. (5).

In order to understand more qualitatively our results, we have performed a comparison to several approximations that have been used in the literature. 


\section{A. Validity of various approximations}

The simplest approximation to the neutron specific heat is to neglect the presence of the nuclear clusters. The specific heat of a Fermi gas is given by

$$
c_{V}^{\mathrm{FG}}(T)=\left(\frac{\pi}{3}\right)^{2 / 3} \frac{m_{n}^{\oplus} T}{\hbar^{2}}\left(\rho_{n}^{G}\right)^{1 / 3},
$$

where $m_{n}^{\oplus}$ is the Skyrme effective mass evaluated at $T=$ 0 for the density of the neutron gas $\rho_{n}^{G}$. Note that for the layers we considered, $m_{n}^{\oplus} \simeq m_{n}$. As shown in Fig. 1, the specific heat given by $(6)$ is very close to the exact result from the band theory.

This striking result can be understood using the semi-classical Extended-Thomas-Fermi expansion method 20]. At lowest order in $\hbar^{2}$, the neutron specific heat is given by

$$
c_{V}^{\mathrm{TF}}(T)=\left(\frac{\pi}{3}\right)^{2 / 3} \frac{T}{\hbar^{2}} \int \frac{\mathrm{d}^{3} \boldsymbol{r}}{V_{\text {cell }}} m_{n}^{\oplus}(\boldsymbol{r}) \rho_{n}(\boldsymbol{r})^{1 / 3} .
$$

We have found that higher order corrections in $\hbar^{2}$ are negligible. The specific heat (7) shown in Fig. 11 for the three selected layers, is very close to the results of band theory. The small effects of the nuclear lattice can now be easily explained by the small volume fraction occupied by the clusters (typically $\sim 10^{-2}-10^{-3}$ ). Indeed in the limit of uniform neutron matter, the Thomas-Fermi specific heat (7) reduces to Eq. (6). A more refined explanation within the full quantum mechanical framework will be given in Sect. II B.

We have also computed the specific heat with the single particle energies $\varepsilon_{\alpha}$ obtained in the Wigner-Seitz approximation. Varying the temperature, we have found a small redistribution of the neutron energies $\varepsilon_{\alpha}$ close to the Fermi energy. Due to the very large number of neutron states, a small redistribution of energies can have a large impact on the total energy and on the entropy. However, it has been found numerically that the entropy being a smooth function of the occupied and unoccupied states, is much less affected by this redistribution than the total energy. We thus have calculated the specific heat using

$$
c_{V}^{\mathrm{WS}}=\left.T \frac{\partial S^{\mathrm{WS}}}{\partial T}\right|_{V}
$$

The entropy density is given by

$$
S^{\mathrm{WS}}=-\frac{1}{V_{\text {cell }}} \sum_{\alpha} g_{\alpha}\left[f_{\alpha} \ln f_{\alpha}+\left(1-f_{\alpha}\right) \ln \left(1-f_{\alpha}\right)\right]
$$

where $g_{\alpha}$ is the degeneracy of the state $\alpha$. The specific heat (8) is shown in Fig. 1. Despite non-linear fluctuations due to the redistribution of neutron states, the results are fairly close to those of the band theory. The best agreement is obtained for the layer with the lowest density, as expected from the domain of validity of the Wigner-Seitz method [14],
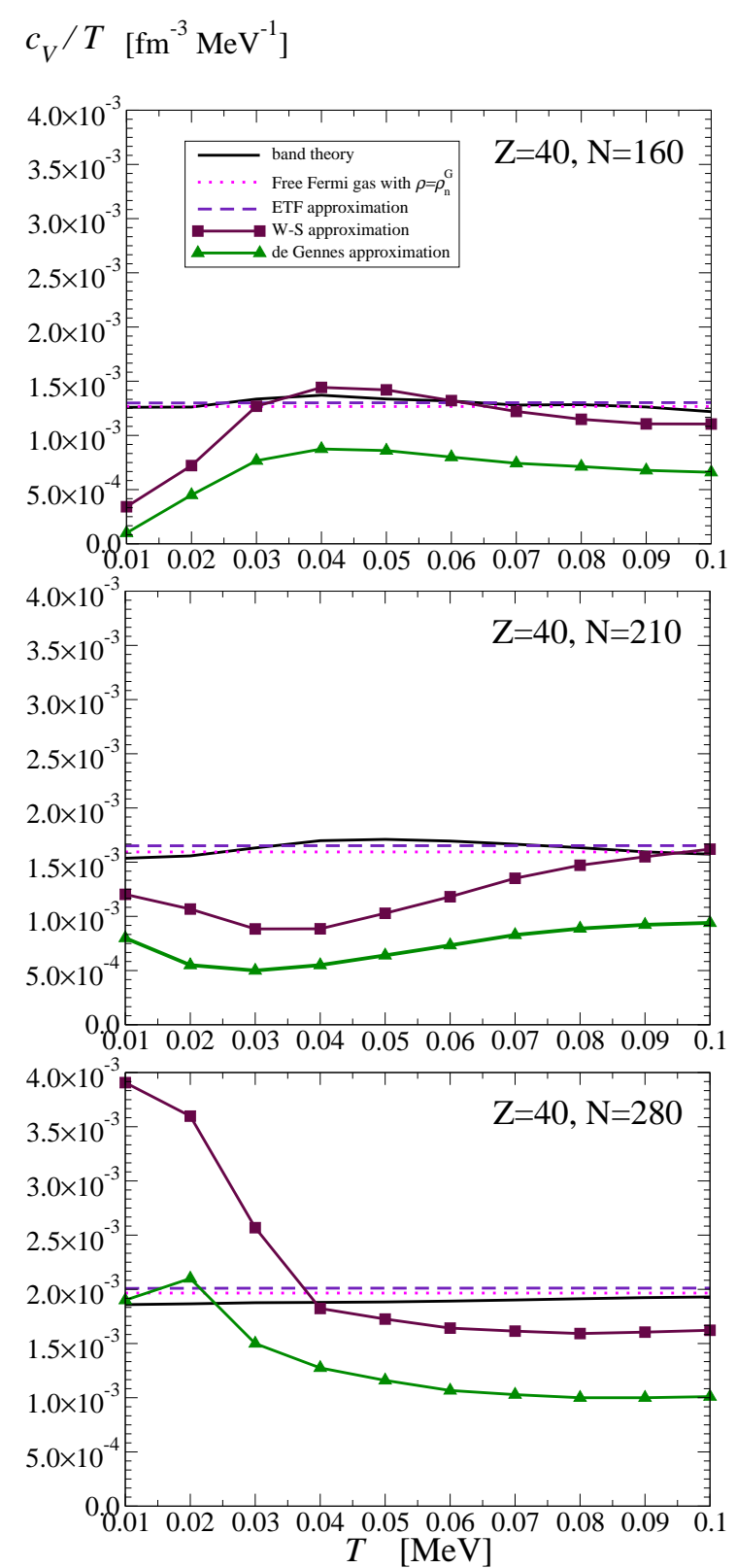

FIG. 1: (color online) Neutron specific heat divided by the temperature in the three shallow layers calculated with the band theory (solid thick line) and with different approximations: free Fermi gas $c_{V}^{\mathrm{FG}}$ (6) (dotted line), Extended-ThomasFermi $c_{V}^{\mathrm{TF}}$ (7) (dashed line), Wigner-Seitz $c_{V}^{\mathrm{WS}}$ (8) (solid line with filled squares) and de Gennes $c_{V}^{\mathrm{DG}}$ (10) (solid line with filled triangle).

We have checked the validity of another approximation, suggested by de Gennes [21] and applied in Ref. [12], consisting in differentiating the entropy (9) with respect to the temperature but assuming that the energies $\varepsilon_{\alpha}$ and the chemical potential $\mu_{n}$ (as well as the pairing gaps in superfluid systems) are independent of $T$. The specific 
heat is then given by

$$
c_{V}^{\mathrm{DG}}(T)=\frac{1}{V_{\text {cell }}} \sum_{\alpha} g_{\alpha} f_{\alpha}\left(1-f_{\alpha}\right)\left(\frac{\varepsilon_{\alpha}-\mu_{n}}{T}\right)^{2} .
$$

As can be seen in Fig. 1, this approximation is valid at very low temperature where the energies and the chemical potential could be taken independent of the temperature but it becomes less and less reliable with increasing temperature.

From the comparison of various approximations to the neutron specific heat, we have found that the Fermi gas model reproduces very well the results of the band theory, while more sophisticated approaches like the WignerSeitz or the de Gennes approximations are less accurate. Does it mean that the unbound neutrons are really "free"?

\section{B. Are unbound neutrons really "free"?}

The present results on the specific heat together with Eq. (5) indicate that the average density of states are close to that of a Fermi gas (as already noticed in a previous paper, see Fig. 6 and 9 of Ref. [14). This conclusion is at first sight surprising since the mean field potential is very deep inside the clusters (about $-70 \mathrm{MeV}$ ). On general grounds, one expects the effects of the nuclear lattice to be negligible whenever the Fermi wavelength of the unbound neutrons $\lambda_{\mathrm{F}}=2 \pi / k_{\mathrm{F}}$ with $k_{\mathrm{F}}=\left(3 \pi^{2} \rho_{n}^{G}\right)^{1 / 3}$, is much larger than the lattice spacing. However this condition is not satisfied for the layers we considered. Using the densities $\rho_{n}^{G}$ given in Table If, we find $\lambda_{\mathrm{F}} \simeq 40.09$, 31.82 and $25.76 \mathrm{fm}$ for $R_{\text {cell }}=49.24,46.33$ and $44.3 \mathrm{fm}$, respectively. One therefore expects the unbound neutrons to be strongly scattered by the clusters.

If the unbound neutrons were really free as suggested by the results on the specific heat, this would imply that their motion is unaffected by the lattice. It is well-known in solid state physics that the interactions between the conduction electrons and the ionic lattice can be taken into account through renormalizating the mass of the electrons. The motion of an electron in a solid, with a wave vector $\boldsymbol{k}$ in a band $\alpha$, can thus be characterized with a dynamical effective mass tensor defined by (ref. [19, p228)

$$
\left.\frac{1}{m_{e}^{\star}}\right|_{i j}=\frac{1}{\hbar^{2}} \frac{\partial^{2} \varepsilon_{\alpha \boldsymbol{k}}^{(e)}}{\partial k_{i} \partial k_{j}}
$$

where $k_{i}, i=1,2,3$ are the Cartesian components of $k$ and $\varepsilon_{\alpha \boldsymbol{k}}^{(e)}$ the electron energy. This concept has been extended to low-energy thermal neutrons propagating in crystals and the corresponding effective mass has been experimentally measured in silicon 22]. In the context of neutron star crust, since the number of unbound neutrons per lattice site can be very large, it is more appropriate to introduce an average effective mass $m_{n}^{\star}$ defined by [15]

$$
m_{n}^{\star}=\rho_{n}^{G} / \mathcal{K}, \quad \mathcal{K}=\frac{1}{3} \sum_{\alpha, i} \int_{\mathrm{F}} \frac{\mathrm{d}^{3} \boldsymbol{k}}{(2 \pi)^{3}} \frac{1}{\hbar^{2}} \frac{\partial^{2} \varepsilon_{\alpha \boldsymbol{k}}}{\partial k_{i} \partial k_{i}},
$$

where the integral is taken over all occupied states. This dynamical effective mass $m_{n}^{\star}$ can be equivalently expressed as

$$
\frac{1}{m_{n}^{\star}}=\frac{1}{\rho_{n}^{G}} \sum_{i} \frac{\partial^{2} U}{\partial p_{n i} \partial p_{n i}},
$$

where $U$ is the energy density of the moving neutrons in the crust frame and $\boldsymbol{p}_{\boldsymbol{n}}$ is the average neutron momentum. This effective mass has implications for neutron star dynamics. For instance it has been shown that a large enough effective mass can trigger a KelvinHelmholtz instability which might explain the origin of pulsar glitches 23.

The dynamical effective mass $m_{n}^{\star}$ has been calculated for the considered layers, using the same numerical method as in Ref. 16. Results are shown in Table II. If the unbound neutrons were free we would have found $m_{n}^{\star} / m_{n}=1$. However, the dynamical effective mass is much larger than the bare neutron mass $m_{n}^{\star} / m_{n}>1$ indicating that the interactions between the neutrons and the clusters are very strong. In the following, we will explain the apparent contradiction with the results obtained previously for the specific heat.

At the beginning of Sect. II, we have shown that the specific heat varies almost linearly with the temperature (see Fig. 1), as expected from Eq. (5). The density of states $g\left(\varepsilon_{\mathrm{F}}\right)$ appearing in Eq. (5) can be expressed as an integral over the Fermi surface $\mathcal{S}_{\mathrm{F}}$,

$$
g\left(\varepsilon_{\mathrm{F}}\right)=\frac{1}{(2 \pi)^{3} \hbar} \sum_{\alpha} \oint_{\mathcal{S}_{\mathrm{F}}} \frac{d \mathcal{S}}{\left|\boldsymbol{v}_{\alpha \boldsymbol{k}}\right|} .
$$

where $\boldsymbol{v}_{\alpha \boldsymbol{k}}=\hbar^{-1} \nabla_{\boldsymbol{k}} \varepsilon_{\alpha \boldsymbol{k}}$ is the group velocity of the unbound neutrons. The presence of nuclear clusters leads to the existence of resonances. As a result, the energy bands of unbound states may be locally flat in k-space thus distorting the Fermi surface 114. The deformation can be estimated by the ratio $\xi_{F}$ of the area of the deformed surface to that of the unperturbed Fermi sphere. Therefore, we have $\mathcal{S}_{\mathrm{F}}=\xi_{\mathrm{F}} 4 \pi k_{\mathrm{F}}^{2}$, where by definition $\xi_{\mathrm{F}}=1$ in the absence of clusters. Numerical calculations show that the nuclear lattice reduces the area of the Fermi surface by more than a factor of 2 in the shallowest layer (see Table III). However since the specific heat is driven by that of a Fermi gas, we can infer from Eq. (14) that in average, the group velocity is changed by the same factor $\xi_{\mathrm{F}}$ compared to that of the Fermi gas.

Likewise the coefficient $\mathcal{K}(12)$ can also be written as an integral over the Fermi surface

$$
\mathcal{K}=\frac{1}{3(2 \pi)^{3} \hbar} \sum_{\alpha} \oint_{\mathcal{S}_{\mathrm{F}}}\left|\boldsymbol{v}_{\alpha \boldsymbol{k}}\right| \mathrm{d} \mathcal{S} .
$$


From the previous discussion, it follows that this coefficient is therefore approximately changed by a factor $\xi_{\mathrm{F}}^{2}$. Eq. (12) then implies that $m_{n}^{\star} \approx m_{n} / \xi_{\mathrm{F}}^{2}$. Inspecting Table 1 shows that the predicted relation between $m_{n}^{\star}$ and $\xi_{\mathrm{F}}$ is only roughly satisfied because $v_{\alpha \boldsymbol{k}}$ varies on the Fermi surface. Nevertheless, this analysis in terms of the topology of the Fermi surface explains why the presence of the nuclear clusters has such a strong impact on the motion of the unbound neutrons but not on their specific heat.

\section{CONCLUSIONS}

Modelling the recently observed thermal relaxation of neutron star crusts requires the knowledge of their thermal properties. In this paper, we have computed the specific heat of the neutron ocean permeating the inner crust at densities below $1.5 \times 10^{12}$ g.cm ${ }^{-3}$, by applying the band theory of solids with the Skyrme SLy4 nucleonnucleon interaction. We have compared the results obtained using different approximations. We have found that for temperatures $T=10^{7}-10^{9} \mathrm{~K}$ relevant to neutron stars, the neutron specific heat is essentially given by that of a Fermi gas. The Thomas-Fermi expression (7) yields nearly undistinguishable results. The specific heat calculated in the Wigner-Seitz approximation agrees reasonably well, while the de Gennes approximation (10) leads to specific heats a factor of 2-3 smaller than those obtained in the band theory.
The results on the specific heat might suggest that the unbound neutrons are not affected by the presence of the nuclear clusters. It is however not true since the dynamical effective mass of the same unbound neutrons is very different from the bare one unvealing strong interactions with the periodic lattice. This apparent paradox can be explained by the fact that the neutron Fermi surface area and the neutron group velocity are both reduced by the same factor in the presence of clusters, leading to a nearly unchanged density of states and specific heat.

TABLE II: For the three shallow layers considered in this paper, are given: the total density $(\rho)$, the dynamical effective mass $\left(m_{n}^{\star} / m_{n}\right)$ and the reduction factor of the Fermi surface area $\left(\xi_{F}\right)$

\begin{tabular}{cccc}
\hline \hline$\rho\left[\mathrm{g} . \mathrm{cm}^{-3}\right]$ & $m_{n}^{\star} / m_{n}$ & $\xi_{F}$ & $\xi_{F}^{2} m_{n}^{\star} / m_{n}$ \\
\hline $6.69 \times 10^{11}$ & 4.0 & 0.44 & 0.77 \\
$1.00 \times 10^{12}$ & 3.6 & 0.49 & 0.86 \\
$1.47 \times 10^{12}$ & 3.2 & 0.52 & 0.87 \\
\hline \hline
\end{tabular}

Acknowledgments

N. C. gratefully acknowledges financial support from a Marie Curie Intra-European grant (contract number MEIF-CT-2005-024660) and from FNRS (Belgium).
[1] P. Haensel, A. Y. Potekhin, D. G. Yakovlev, Neutron Stars 1: Equation of state and structure, Springer (2007).

[2] U. Lombardo, in "Nuclear Methods and the Nuclear Equation of State, Int. Rev. of Nucl. Physics, Vol. 9, M. Baldo Eds. (World-Scientific, Singapore, 1999) p458.

[3] T. Strohmayer, L. Bildsten in "Compact Stellar X-Ray Sources, eds W. Lewin and M. van der Klis, Cambridge Astrophysics Series 39 (2006), p113.

[4] M. Grasso, E. Khan, J. Margueron, N. Van Giai, Nucl. Phys. A 807 (2008), 1.

[5] E. M. Cackett, R. Wijnands, M. Linares, J. M. Miller, J. Homan, W. H. G. Lewin, Mon.Not.Roy.Astron.Soc. 372 (2006) 479.

[6] R. E. Rutledge, L. Bildsten, E. F. Brown, G. Pavlov, V. E. Zavlin, G. Ushomirsky, Astrophys. J. 580 (2002) 413.

[7] P. S. Shternin, D. G. Yakovlev, P. Haensel, A. Y. Potekhin, Mon.Not.Roy.Astron.Soc.Lett. 382 (2007) L43.

[8] Lattimer, Van Riper, Prakash, Prakash, Astrophys. Jour. 425 (1994), 802.

[9] O.Y. Gnedin, D.G. Yakovlev, A.Y. Potekhin, Mon.Not.Roy.Astron.Soc. 324 (2001) 725.

[10] Levenfish and Yakovlev, Astron. Rep. 38 (1994) 247.

[11] P. M. Pizzochero, F. Barranco, E. Vigezzi, R. A. Broglia, Astrophys. J. 569 (2002) 381.

[12] C. Monrozeau, J. Margueron, N. Sandulescu, Phys. Rev.
C 75 (2007) 065807.

[13] E. Khan, N. Sandulescu and N. Van Giai, Phys. Rev. C 71 (2005) 042801(R).

[14] N. Chamel, S. Naimi, E. Khan, J. Margueron, Phys. Rev. C75 (2007) 055806.

[15] B. Carter, N. Chamel, P. Haensel, Nucl. Phys. A 748 (2005) 675.

[16] N. Chamel, Nucl.Phys. A747 (2005) 109; N. Chamel, Nucl.Phys. A773 (2006) 263.

[17] J.W. Negele and D. Vautherin, Nucl. Phys. A207 (1973) 298.

[18] E. Chabanat, P. Bonche, P. Haensel, J. Meyer, R. Schaeffer, Nucl. Phys. A635 231-256 (1998) ; Erratum, Nucl. Phys. A643 441 (1998).

[19] N. W. Ashcroft and N. D. Mermin, Solid State Physics, Holt, Rinehart and Winston (1976).

[20] M. Onsi, A. K. Dutta, H. Chatri, S. Goriely, N. Chamel, J. M. Pearson, Phys. Rev. C 77 (2008), 065805.

[21] P. G. de Gennes, Superconductivity of Metals and Alloys, W.A. Benjamin inc., New York Amsterdam (1966), p129.

[22] A. Zeilinger, C. G. Shull, M. A. Horne, and K. D. Finkelstein, Phys. Rev. Lett. 57 (1986), 3089-3092 ; K. Raum, M. Koellner, A. Zeilinger, M. Arif, R. Gahler, Phys. Rev. Lett. 74 (1995), 2859-2862.

[23] N. Andersson, G. L. Comer, R. Prix, Phys. Rev. Lett. 90, 091101 (2003). 